Pacific

Journal of

Mathematics

\title{
RADIAL LIMITS OF BOUNDED NONPARAMETRIC PRESCRIBED MEAN CURVATURE SURFACES
}

Mozhgan (NoRa) Entekhabi AND Kirk E. LANCASter 


\title{
RADIAL LIMITS OF BOUNDED NONPARAMETRIC PRESCRIBED MEAN CURVATURE SURFACES
}

\author{
Mozhgan (Nora) EnTEKhabi And Kirk E. LANCASter
}

Dedicated to the memory of Alan Ross Elcrat

Consider a solution $f \in C^{2}(\Omega)$ of a prescribed mean curvature equation

$$
\operatorname{div} \frac{\nabla f}{\sqrt{1+|\nabla f|^{2}}}=2 H(x, f) \text { in } \Omega,
$$

where $\Omega \subset \mathbb{R}^{2}$ is a domain whose boundary has a corner at $\mathcal{O}=(0,0) \in \partial \Omega$. If $\sup _{x \in \Omega}|f(x)|$ and $\sup _{x \in \Omega}|H(x, f(x))|$ are both finite and $\Omega$ has a reentrant corner at $\mathcal{O}$, then the (nontangential) radial limits of $f$ at $\mathcal{O}$,

$$
R f(\theta):=\lim _{r \downarrow 0} f(r \cos \theta, r \sin \theta),
$$

are shown to exist, independent of the boundary behavior of $f$ on $\partial \Omega$, and to have a specific type of behavior. If $\sup _{x \in \Omega}|f(x)|$ and $\sup _{x \in \Omega}|H(x, f(x))|$ are both finite and the trace of $f$ on one side has a limit at $\mathcal{O}$, then the (nontangential) radial limits of $f$ at $\mathcal{O}$ exist, the tangential radial limit of $f$ at $\mathcal{O}$ from one side exists and the radial limits have a specific type of behavior.

\section{Introduction and statement of main theorems}

Consider the prescribed mean curvature equation

$$
N f=2 H(\cdot, f) \text { in } \Omega,
$$

where $\Omega$ is a domain in $\mathbb{R}^{2}$ whose boundary has a corner at $\mathcal{O} \in \partial \Omega, N f=$ $\nabla \cdot T f=\operatorname{div}(T f), T f=(\nabla f) / \sqrt{1+|\nabla f|^{2}}, H: \Omega \times \mathbb{R} \rightarrow \mathbb{R}$ and $H$ satisfies one of the conditions which guarantees that "cusp solutions" (e.g., [Lancaster and Siegel 1996a, §5; 1996b]) do not exist; for example, $H(\boldsymbol{x}, t)$ is strictly increasing in $t$ for each $\boldsymbol{x}$ or is real-analytic (e.g., constant). We will assume $\mathcal{O}=(0,0)$. Let $\Omega^{*}=\Omega \cap B_{\delta^{*}}(\mathcal{O})$, where $B_{\delta^{*}}(\mathcal{O})$ is the ball in $\mathbb{R}^{2}$ of radius $\delta^{*}$ about $\mathcal{O}$. Polar coordinates relative to $\mathcal{O}$ will be denoted by $r$ and $\theta$. We assume that $\partial \Omega$ is piecewise smooth and there exists $\alpha \in(0, \pi)$ such that $\partial \Omega \cap B_{\delta^{*}}(\mathcal{O})$ consists of

MSC2010: 35B40, 53A10.

Keywords: prescribed mean curvature, radial limits. 


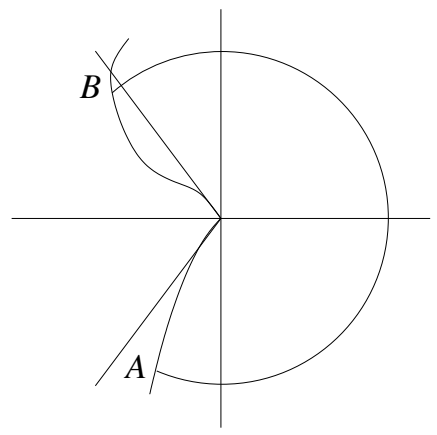

Figure 1. The domain $\Omega^{*}$.

two $\operatorname{arcs} \partial^{+} \Omega^{*}$ and $\partial^{-} \Omega^{*}$, whose tangent lines approach the lines $L^{+}: \theta=\alpha$ and $L^{-}: \theta=-\alpha$, respectively, as the point $\mathcal{O}$ is approached (see Figure 1 of [Lancaster and Siegel 1997] or Figure 1).

Suppose

$$
\sup _{x \in \Omega}|f(x)|<\infty \quad \text { and } \quad \sup _{x \in \Omega}|H(x, f(x))|<\infty .
$$

We shall prove

Theorem 1. Let $f \in C^{2}(\Omega)$ satisfy (1) and suppose (2) holds and $\alpha \in(\pi / 2, \pi)$. Then for each $\theta \in(-\alpha, \alpha)$,

$$
R f(\theta):=\lim _{r \downarrow 0} f(r \cos \theta, r \sin \theta)
$$

exists and $R f(\cdot)$ is a continuous function on $(-\alpha, \alpha)$ which behaves in one of the following ways:

(i) $R f:(-\alpha, \alpha) \rightarrow \mathbb{R}$ is a constant function (so $f$ has a nontangential limit at $\mathcal{O}$ ).

(ii) There exist $\alpha_{1}$ and $\alpha_{2}$ so that $-\alpha \leq \alpha_{1}<\alpha_{2} \leq \alpha$ and $R f$ is constant on $\left(-\alpha, \alpha_{1}\right]$ and $\left[\alpha_{2}, \alpha\right)$ and strictly increasing or strictly decreasing on $\left(\alpha_{1}, \alpha_{2}\right)$.

(iii) There exist $\alpha_{1}, \alpha_{L}, \alpha_{R}, \alpha_{2}$ so that $-\alpha \leq \alpha_{1}<\alpha_{L}<\alpha_{R}<\alpha_{2} \leq \alpha, \alpha_{R}=$ $\alpha_{L}+\pi$, and $R f$ is constant on $\left(-\alpha, \alpha_{1}\right],\left[\alpha_{L}, \alpha_{R}\right]$, and $\left[\alpha_{2}, \alpha\right)$ and either strictly increasing on $\left(\alpha_{1}, \alpha_{L}\right]$ and strictly decreasing on $\left[\alpha_{R}, \alpha_{2}\right)$ or strictly decreasing on $\left(\alpha_{1}, \alpha_{L}\right]$ and strictly increasing on $\left[\alpha_{R}, \alpha_{2}\right)$.

At a convex corner (i.e., $\alpha \in(0, \pi / 2])$, Theorem 1 is not applicable. The additional assumption that the trace of $f$ on one side (e.g., $\partial^{-} \Omega^{*}$ ) has a limit at $\mathcal{O}$ implies the radial limits of $f$ exist.

Theorem 2. Let $f \in C^{2}(\Omega) \cap C^{0}\left(\Omega \cup \partial^{-} \Omega^{*} \backslash\{\mathcal{O}\}\right)$ satisfy (1). Suppose (2) holds and $m=\lim _{\partial^{-} \Omega^{*} \ni x \rightarrow \mathcal{O}} f(\boldsymbol{x})$ exists. Then for each $\theta \in(-\alpha, \alpha), R f(\theta)$ exists and $R f(\cdot)$ is a continuous function on $[-\alpha, \alpha)$, where $R f(-\alpha):=m$. If $\alpha \in(0, \pi / 2]$, 
$R f$ can behave as in (i) or (ii) in Theorem 1. If $\alpha \in(\pi / 2, \pi), R f$ can behave as in (i), (ii) or (iii) in Theorem 1.

The conclusions of these theorems were first obtained in [Lancaster 1985] for minimal surfaces satisfying Dirichlet boundary conditions and then for nonparametric prescribed mean curvature surfaces satisfying Dirichlet [Elcrat and Lancaster 1986; Lancaster 1988] or contact angle [Lancaster and Siegel 1996a] boundary conditions; see also [Jin and Lancaster 1997; Lancaster 1991]. Notice that Theorem 1 applies to a solution of a capillary surface problem whose domain has a reentrant corner even when the contact angle equals 0 and/or $\pi$ on some (or all) of $\partial \Omega^{*}$.

Remark. The assumption that $\Omega$ has a reentrant corner at $\mathcal{O} \in \partial \Omega$ or that the trace of $f$ from one side of $\partial \Omega$ is continuous at $\mathcal{O}$ is critical here; the nonexistence of radial limits at $(1,0)$ when $\Omega=B_{1}(\mathcal{O})$ and the boundary data is symmetric with respect to the horizontal axis is demonistrated in [Lancaster 1989] and in Theorem 3 of [Lancaster and Siegel 1996a]. In [Lancaster 1987], it was conjectured that the existence of radial limits at corners for bounded solutions of Dirichlet problems for the minimal surface equation in $\mathbb{R}^{2}$, independent of boundary conditions. Although [Lancaster 1989] proved this conjecture false, Theorems 1 and 2 show it is true in many cases.

\section{Proof of Theorem 1}

Since $f \in C^{2}(\Omega)$ (and so in $C^{0}(\Omega)$ ), we may assume that $f$ is uniformly continuous on $\left\{\boldsymbol{x} \in \Omega^{*}:|\boldsymbol{x}|>\delta\right\}$ for each $\delta \in\left(0, \delta^{*}\right)$; if this is not true, we may replace $\Omega$ with $U, U \subset \Omega$, such that $\partial \Omega \cap \partial U=\{\mathcal{O}\}$ and $\partial U \cap B_{\delta^{*}}(\mathcal{O})$ consists of two arcs $\partial^{+} U$ and $\partial^{-} U$, whose tangent lines approach the lines $L^{+}: \theta=\alpha$ and $L^{-}: \theta=-\alpha$, respectively, as the point $\mathcal{O}$ is approached. Set

$$
S_{0}^{*}=\left\{(\boldsymbol{x}, f(\boldsymbol{x})): \boldsymbol{x} \in \Omega^{*}\right\} \quad \text { and } \quad \Gamma_{0}^{*}=\left\{(\boldsymbol{x}, f(\boldsymbol{x})): \boldsymbol{x} \in \partial \Omega^{*} \backslash\{\mathcal{O}\}\right\} ;
$$

the points where $\partial B_{\delta^{*}}(\mathcal{O})$ intersect $\partial \Omega$ are labeled $A \in \partial^{-} \Omega^{*}$ and $B \in \partial^{+} \Omega^{*}$. From the calculation on page 170 of [Lancaster and Siegel 1996a], we see that the area of $S_{0}^{*}$ is finite; let $M_{0}$ denote this area. For $\delta \in(0,1)$, set

$$
p(\delta)=\sqrt{\frac{8 \pi M_{0}}{\ln (1 / \delta)}} .
$$

Let $E=\left\{(u, v): u^{2}+v^{2}<1\right\}$. As in [Elcrat and Lancaster 1986; Lancaster and Siegel 1996a], there is a parametric description of the surface $S_{0}^{*}$,

$$
Y(u, v)=(a(u, v), b(u, v), c(u, v)) \in C^{2}\left(E: \mathbb{R}^{3}\right),
$$

which has the following properties: 
$\left(a_{1}\right) Y$ is a diffeomorphism of $E$ onto $S_{0}^{*}$.

$\left(a_{2}\right)$ Set $G(u, v)=(a(u, v), b(u, v)),(u, v) \in E$. Then $G \in C^{0}\left(\bar{E}: \mathbb{R}^{2}\right)$.

$\left(a_{3}\right)$ Let $\sigma=G^{-1}\left(\partial \Omega^{*} \backslash\{\mathcal{O}\}\right)$; then $\sigma$ is a connected arc of $\partial E$ and $Y$ maps $\sigma$ strictly monotonically onto $\Gamma_{0}^{*}$. We may assume the endpoints of $\sigma$ are $\boldsymbol{o}_{1}$ and $\boldsymbol{o}_{2}$ and there exist points $\boldsymbol{a}, \boldsymbol{b} \in \sigma$ such that $G(\boldsymbol{a})=A, G(\boldsymbol{b})=B, G$ maps the (open) arc $\boldsymbol{o}_{1} \boldsymbol{b}$ onto $\partial^{+} \Omega$, and $G$ maps the (open) arc $\boldsymbol{o}_{2} \boldsymbol{a}$ onto $\partial^{-} \Omega$. (Note that $\boldsymbol{o}_{1}$ and $\boldsymbol{o}_{2}$ are not assumed to be distinct at this point; Figures $4 \mathrm{a}$ and $4 \mathrm{~b}$ of [Lancaster and Siegel 1997] illustrate this situation.)

(a4) $Y$ is conformal on $E: Y_{u} \cdot Y_{v}=0, Y_{u} \cdot Y_{u}=Y_{v} \cdot Y_{v}$ on $E$.

(as) $\Delta Y:=Y_{u u}+Y_{v v}=H(Y) Y_{u} \times Y_{v}$ on $E$.

Here by the (open) $\operatorname{arcs} \boldsymbol{o}_{1} \boldsymbol{b}$ and $\boldsymbol{o}_{2} \boldsymbol{a}$ we mean the component of $\partial E \backslash\left\{\boldsymbol{o}_{1}, \boldsymbol{b}\right\}$ which does not contain $\boldsymbol{a}$ and the component of $\partial E \backslash\left\{\boldsymbol{o}_{2}, \boldsymbol{a}\right\}$ which does not contain $\boldsymbol{b}$ respectively. Let $\sigma_{0}=\partial E \backslash \sigma$.

There are two cases we wish to consider:

(A) $\boldsymbol{o}_{1}=\boldsymbol{o}_{2}$.

(B) $\boldsymbol{o}_{1} \neq \boldsymbol{o}_{2}$.

These correspond to Cases 5 and 3 respectively in Step 1 of the proof of Theorem 1 in [Lancaster and Siegel 1996a]. Let us first assume that (A) holds and set $\boldsymbol{o}=\boldsymbol{o}_{1}=\boldsymbol{o}_{2}$. Let $h$ denote a function on the annulus $\mathcal{A}=\left\{\boldsymbol{x}: r_{1} \leq|\boldsymbol{x}| \leq r_{2}\right\}$ which vanishes on the circle $|\boldsymbol{x}|=r_{2}$ and whose graph is an unduloid surface with constant mean curvature $-H_{0}$ which becomes vertical at $|\boldsymbol{x}|=r_{1}$ and at $|\boldsymbol{x}|=r_{2}$ (see Figure 2) for suitable $r_{1}<r_{2}$ (e.g., [Lancaster and Siegel 1996a, pp. 170-171]). Let $q$ denote the modulus of continuity of $h$ (i.e., $\left|h\left(\boldsymbol{x}_{1}\right)-h\left(\boldsymbol{x}_{2}\right)\right| \leq q\left(\left|\boldsymbol{x}_{1}-\boldsymbol{x}_{2}\right|\right)$ ).

For each $\boldsymbol{p} \in \mathbb{R}^{2}$ with $|\boldsymbol{p}|=r_{1}$, set $\mathcal{A}(\boldsymbol{p})=\left\{\boldsymbol{x}: r_{1} \leq|\boldsymbol{x}-\boldsymbol{p}| \leq r_{2}\right\}$ and define $h_{\boldsymbol{p}}: \mathcal{A}(\boldsymbol{p}) \rightarrow \mathbb{R}$ by $h_{\boldsymbol{p}}(\boldsymbol{x})=h(\boldsymbol{x}-\boldsymbol{p})$.

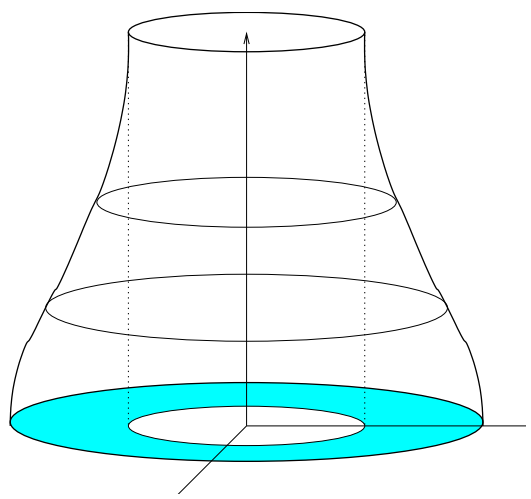

Figure 2. The graph of $h$ over $\mathcal{A}$. 


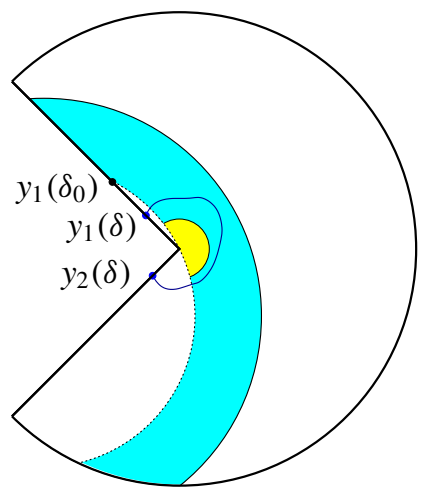

Figure 3. $\Omega^{*} \cap \mathcal{A}\left(\boldsymbol{p}_{1}\right), C_{\rho(\delta)}^{\prime}$ (blue curve), $B_{\eta(\delta)}(\mathcal{O}) \cap \mathcal{A}\left(\boldsymbol{p}_{1}\right)$ (yellow).

For $r>0$, set $B_{r}=\{\boldsymbol{u} \in \bar{E}:|\boldsymbol{u}-\boldsymbol{o}|<r\}, C_{r}=\{\boldsymbol{u} \in \bar{E}:|\boldsymbol{u}-\boldsymbol{o}|=r\}$ and let $l_{r}$ be the length of the image curve $Y\left(C_{r}\right)$; also let $C_{r}^{\prime}=G\left(C_{r}\right)$ and $B_{r}^{\prime}=G\left(B_{r}\right)$. From the Courant-Lebesgue lemma (e.g., [Courant 1950, Lemma 3.1]), we see that for each $\delta \in(0,1)$, there exists a $\rho=\rho(\delta) \in(\delta, \sqrt{\delta})$ such that the arclength $l_{\rho}$ of $Y\left(C_{\rho}\right)$ is less than $p(\delta)$. For $\delta>0$, let $k(\delta)=\inf _{\boldsymbol{u} \in C_{\rho(\delta)}} c(\boldsymbol{u})=\inf _{\boldsymbol{x} \in C_{\rho(\delta)}^{\prime}} f(\boldsymbol{x})$ and $m(\delta)=\sup _{\boldsymbol{u} \in C_{\rho(\delta)}} c(\boldsymbol{u})=\sup _{\boldsymbol{x} \in C_{\rho(\delta)}^{\prime}} f(\boldsymbol{x})$; notice that $m(\delta)-k(\delta) \leq l_{\rho}^{\rho(\delta)}<p(\delta)$.

For each $\delta \in(0,1)$ with $\sqrt{\delta}<\min \{|\boldsymbol{o}-\boldsymbol{a}|,|\boldsymbol{o}-\boldsymbol{b}|\}$, there are two points in $C_{\rho(\delta)} \cap \partial E$; we denote these points as $\boldsymbol{e}_{1}(\delta) \in \boldsymbol{o b}$ and $\boldsymbol{e}_{2}(\delta) \in \boldsymbol{o a}$ and set $\boldsymbol{y}_{1}(\delta)=$ $G\left(\boldsymbol{e}_{1}(\delta)\right)$ and $\boldsymbol{y}_{2}(\delta)=G\left(\boldsymbol{e}_{2}(\delta)\right)$. Notice that $C_{\rho(\delta)}^{\prime}$ is a curve in $\bar{\Omega}$ which joins $\boldsymbol{y}_{1} \in \partial^{+} \Omega^{*}$ and $\boldsymbol{y}_{2} \in \partial^{-} \Omega^{*}$ and $\partial \Omega \cap C_{\rho(\delta)}^{\prime} \backslash\left\{\boldsymbol{y}_{1}, \boldsymbol{y}_{2}\right\}=\varnothing$; therefore there exists $\eta=\eta(\delta)>0$ such that $B_{\eta(\delta)}(\mathcal{O})=\{\boldsymbol{x} \in \Omega:|\boldsymbol{x}|<\eta(\delta)\} \subset B_{\rho(\delta)}^{\prime}$ (see Figure 3).

Fix $\delta_{0} \in\left(0, \delta^{*}\right)$ with $\sqrt{\delta_{0}}<\min \{|\boldsymbol{o}-\boldsymbol{a}|,|\boldsymbol{o}-\boldsymbol{b}|\}$. Let $\boldsymbol{p}_{1} \in \mathbb{R}^{2}$ satisfy $\left|\boldsymbol{p}_{1}\right|=r_{1}$ and $\left|\boldsymbol{p}_{1}-\boldsymbol{y}_{1}\left(\delta_{0}\right)\right|=r_{1}$ such that $\boldsymbol{p}_{1}$ lies below (and to the left of) the line through $\mathcal{O}$ and $\boldsymbol{y}_{1}\left(\delta_{0}\right)$. Let $\boldsymbol{p}_{2} \in \mathbb{R}^{2}$ satisfy $\left|\boldsymbol{p}_{2}\right|=r_{1}$ and $\left|\boldsymbol{p}_{2}-\boldsymbol{y}_{2}\left(\delta_{0}\right)\right|=r_{1}$ such that $\boldsymbol{p}_{2}$ lies above (and to the left of) the line through $\mathcal{O}$ and $\boldsymbol{y}_{2}\left(\delta_{0}\right)$. Set $\Omega_{0}=\left\{\boldsymbol{x} \in \Omega^{*}\right.$ : $\left.\left|\boldsymbol{x}-\boldsymbol{p}_{1}\right|>r_{1}\right\} \cup\left\{\boldsymbol{x} \in \Omega^{*}:\left|\boldsymbol{x}-\boldsymbol{p}_{2}\right|>r_{1}\right\}$ (see Figure 4).

Claim. $f$ is uniformly continuous on $\Omega_{0}$.

Proof. Let $\epsilon>0$. Choose $\delta \in\left(0, \delta_{0}\right)$ such that $p(\delta)+q(p(\delta))<\frac{1}{4} \epsilon$ and $p(\delta)<r_{2}-r_{1}$. Pick a point $\boldsymbol{w} \in C_{\rho(\delta)}^{\prime}$ and define $b_{j}^{ \pm}: \mathcal{A}\left(\boldsymbol{p}_{j}\right) \rightarrow \mathbb{R}$ by

$$
b_{j}^{ \pm}(\boldsymbol{x})=f(\boldsymbol{w}) \pm p(\delta) \pm h_{\boldsymbol{p}_{j}}(\boldsymbol{x}), \quad \boldsymbol{x} \in \mathcal{A}\left(\boldsymbol{p}_{j}\right)
$$

for $j \in\{1,2\}$. Notice that

$$
b_{j}^{-}(\boldsymbol{x})<f(\boldsymbol{x})<b_{j}^{+}(\boldsymbol{x}) \quad \text { for } \boldsymbol{x} \in B_{\rho(\delta)}^{\prime} \cap \mathcal{A}\left(\boldsymbol{p}_{j}\right), \quad j \in\{1,2\} .
$$




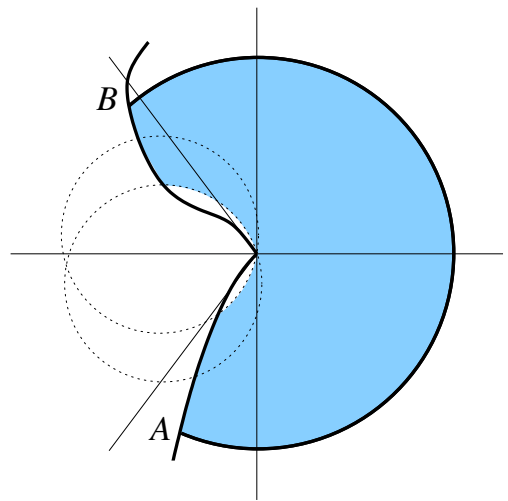

Figure 4. $\Omega_{0}$.

If $\boldsymbol{x}_{1}, \boldsymbol{x}_{2} \in \Omega_{0}$ satisfy $\left|\boldsymbol{x}_{1}\right|<\eta(\delta)$ and $\left|\boldsymbol{x}_{2}\right|<\eta(\delta)$, then there exist $\boldsymbol{x}_{3} \in \mathcal{A}\left(\boldsymbol{p}_{1}\right) \cap$ $\mathcal{A}\left(\boldsymbol{p}_{2}\right)$ with $\left|\boldsymbol{x}_{3}\right|<\eta(\delta)$ such that $\left|\boldsymbol{x}_{1}-\boldsymbol{x}_{3}\right|<\eta(\delta)$ and $\left|\boldsymbol{x}_{2}-\boldsymbol{x}_{3}\right|<\eta(\delta)$ and so

$$
\left|f\left(\boldsymbol{x}_{1}\right)-f\left(\boldsymbol{x}_{2}\right)\right| \leq\left|f\left(\boldsymbol{x}_{1}\right)-f\left(\boldsymbol{x}_{3}\right)\right|+\left|f\left(\boldsymbol{x}_{1}\right)-f\left(\boldsymbol{x}_{3}\right)\right|<4 p(\delta)+4 q(p(\delta))<\epsilon .
$$

Since $f$ is uniformly continuous on $\left\{\boldsymbol{x} \in \Omega^{*}:|\boldsymbol{x}| \geq \frac{1}{2} \eta(\delta)\right\}$, there exists a $\lambda>0$ such that if $\boldsymbol{x}_{1}, \boldsymbol{x}_{2} \in \Omega^{*}$ satisfy $\left|\boldsymbol{x}_{1}-\boldsymbol{x}_{2}\right| \geq \frac{1}{2} \eta(\delta)$ and $\left|\boldsymbol{x}_{1}-\boldsymbol{x}_{2}\right|<\lambda$, then $\left|f\left(\boldsymbol{x}_{1}\right)-f\left(\boldsymbol{x}_{2}\right)\right|<\epsilon$. Now set $d=d(\epsilon)=\min \left\{\lambda, \frac{1}{2} \eta(\delta)\right\}$. If $\boldsymbol{x}_{1}, \boldsymbol{x}_{2} \in \Omega_{0}, \mid \boldsymbol{x}_{1}-$ $\boldsymbol{x}_{2} \mid<d(\epsilon) \leq \frac{1}{2} \eta(\delta)$ and $\left|\boldsymbol{x}_{1}\right|<\frac{1}{2} \eta(\delta)$, then $\left|\boldsymbol{x}_{1}\right|<\eta(\delta)$ and $\left|\boldsymbol{x}_{2}\right|<\eta(\delta)$; hence $\left|f\left(x_{1}\right)-f\left(x_{2}\right)\right|<\epsilon$ by (4). Next, if $x_{1}, x_{2} \in \Omega_{0},\left|x_{1}-x_{2}\right|<d(\epsilon) \leq \lambda,\left|x_{1}\right| \geq \frac{1}{2} \eta(\delta)$ and $\left|x_{2}\right| \geq \frac{1}{2} \eta(\delta)$, then $\left|f\left(x_{1}\right)-f\left(x_{2}\right)\right|<\epsilon$. Therefore, for all $\boldsymbol{x}_{1}, \boldsymbol{x}_{2} \in \Omega_{0}$ with $\left|x_{1}-x_{2}\right|<d(\epsilon)$, we have $\left|f\left(x_{1}\right)-f\left(x_{2}\right)\right|<\epsilon$.

If $\left\{\left(r \cos \left(\theta^{-}\left(\delta_{0}\right)\right), r \sin \left(\theta^{-}\left(\delta_{0}\right)\right)\right): r \geq 0\right\}$ is the tangent ray to $\partial \mathcal{A}\left(\boldsymbol{p}_{2}\right)$ at $\mathcal{O}$, $\left\{\left(r \cos \left(\theta^{+}\left(\delta_{0}\right)\right), r \sin \left(\theta^{+}\left(\delta_{0}\right)\right)\right): r \geq 0\right\}$ is the tangent ray to $\partial \mathcal{A}\left(\boldsymbol{p}_{1}\right)$ at $\mathcal{O}$ and $\theta^{-}\left(\delta_{0}\right), \theta^{+}\left(\delta_{0}\right) \in(-\alpha, \alpha)$, then it follows from the claim that $f \in C^{0}\left(\bar{\Omega}_{0}\right)$, the radial limits $R f(\theta)$ of $f$ at $\mathcal{O}$ exist for $\theta \in\left[\theta^{-}\left(\delta_{0}\right), \theta^{+}\left(\delta_{0}\right)\right]$ and the radial limits are identical (i.e., $R f(\theta)=f(\mathcal{O})$ for all $\theta \in\left[\theta^{-}\left(\delta_{0}\right), \theta^{+}\left(\delta_{0}\right)\right]$.) Since

$$
\lim _{\delta_{0} \downarrow 0} \theta^{-}\left(\delta_{0}\right)=-\alpha \quad \text { and } \quad \lim _{\delta_{0} \downarrow 0} \theta^{+}\left(\delta_{0}\right)=\alpha,
$$

Theorem 1 is proven in this case.

Next assume that (B) holds. For $r>0$ and $j \in\{1,2\}$, set $B_{r}^{j}=\left\{\boldsymbol{u} \in \bar{E}:\left|\boldsymbol{u}-\boldsymbol{o}_{j}\right|<r\right\}$, $C_{r}^{j}=\left\{\boldsymbol{u} \in \bar{E}:\left|\boldsymbol{u}-\boldsymbol{o}_{j}\right|=r\right\}$, and let $l_{r}^{j}$ be the length of the image curve $Y\left(C_{r}^{j}\right)$; also let $C_{r}^{j, \prime}=G\left(C_{r}^{j}\right)$ and $B_{r}^{j, \prime}=G\left(B_{r}^{j}\right)$. From the Courant-Lebesgue lemma, we see that for each $\delta \in(0,1)$ and $j \in\{1,2\}$, there exists a $\rho_{j}=\rho_{j}(\delta) \in(\delta, \sqrt{\delta})$ such that the arclength $l_{j, \rho}$ of $Y\left(C_{\rho_{j}}^{j}\right)$ is less than $p(\delta)$.

We will only consider $\delta \leq \delta_{0}$, where $\delta_{0}$ is small enough that the endpoints of $C_{\rho_{j}(\delta)}^{j}$ lie on $\sigma_{0} \cup \sigma_{N}^{j}$ for $j \in\{1,2\}$ and $C_{\sqrt{\delta_{0}}}^{1} \cap C_{\sqrt{\delta_{0}}}^{2}=\varnothing$, where $\sigma_{N}^{1}=\boldsymbol{o}_{1} \boldsymbol{b}$ and 


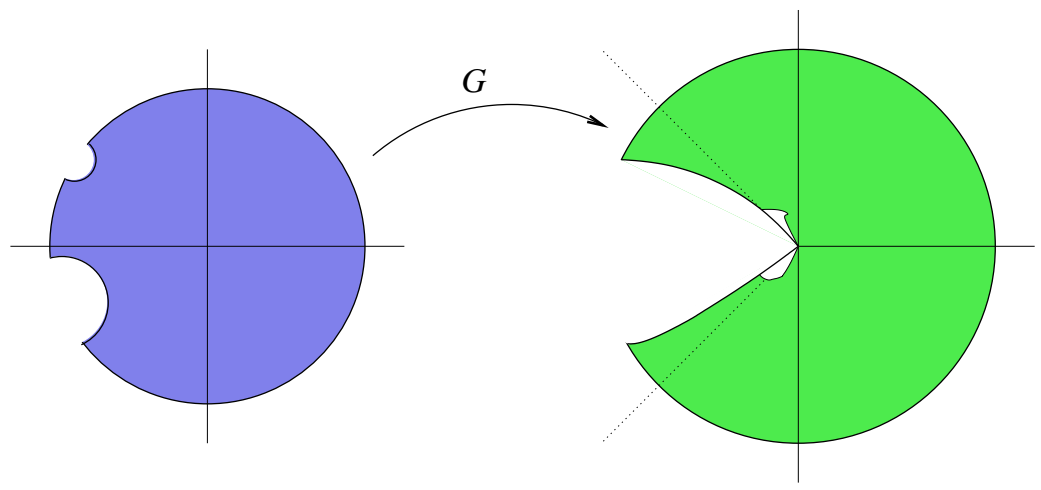

Figure 5. $E \backslash\left(\overline{B_{\rho_{1}(\delta)}^{1}} \cup \overline{B_{\rho_{2}(\delta)}^{2}}\right)$ and $\Omega_{1}$.

$\sigma_{N}^{2}=\boldsymbol{o}_{2} \boldsymbol{a}$. For each $\delta \in\left(0, \delta_{0}\right)$, the fact that $l_{j, \rho_{j}(\delta)}$ is finite for $j \in\{1,2\}$ implies that

$$
\lim _{C_{\rho_{j}(\delta)}^{j,} \ni \boldsymbol{x} \rightarrow \mathcal{O}} f(\boldsymbol{x}) \quad \text { exists for } j \in\{1,2\} .
$$

If we set $\Omega_{1}=G\left(E \backslash\left(\overline{B_{\rho_{1}(\delta)}^{1}} \cup \overline{B_{\rho_{2}(\delta)}^{2}}\right)\right)$ and define $\phi: \partial \Omega_{1} \rightarrow \mathbb{R}$ by $\phi=f$, then $\phi$ has (at worst) a jump discontinuity at $\mathcal{O}$. If we consider $\phi$ to be the Dirichlet data for the boundary value problem

$$
\begin{aligned}
\operatorname{div}(T h) & =2 H(\cdot, f) & & \text { in } \Omega_{1}, \\
h & =\phi & & \text { on } \partial \Omega_{1} \backslash\{\mathcal{O}\},
\end{aligned}
$$

then $h=f$ is the unique solution of this boundary value problem and so we may parametrize the graph of $f$ over $\Omega_{1}$ in isothermal coordinates as above and the arguments in [Elcrat and Lancaster 1986; Lancaster 1988; Lancaster and Siegel 1996a] can be used to show that $c$ is uniformly continuous on $\Omega_{1}$ and so extends to be continuous on $\bar{\Omega}_{1}$. That is, let $k: E \backslash\left(\overline{B_{\rho_{1}(\delta)}^{1}} \cup \overline{B_{\rho_{2}(\delta)}^{2}}\right) \rightarrow E$ be a conformal map. From the works just cited we see that $c \circ k^{-1} \in C^{0}(\bar{E})$ and so $c \in C^{0}\left(\overline{E \backslash\left(B_{\rho_{1}(\delta)}^{1} \cup B_{\rho_{2}(\delta)}^{2}\right.}\right)$. Since

$$
\bigcup_{\delta \in(0,1)}\left(E \backslash\left(B_{\rho_{1}(\delta)}^{1} \cup B_{\rho_{2}(\delta)}^{2}\right)\right)=E,
$$

we see $c \in C^{0}\left(\bar{E} \backslash\left\{\boldsymbol{o}_{1}, \boldsymbol{o}_{2}\right\}\right)$.

As at the end of Step 1 of the proof of Theorem 1 of [Lancaster and Siegel 1996a], we define $X: B \rightarrow \mathbb{R}^{3}$ by $X=Y \circ g$ and $K: B \rightarrow \mathbb{R}^{2}$ by $K=G \circ g$, where $B=\{(u, v) \in$ $\left.\mathbb{R}^{2}: u^{2}+v^{2}<1, v>0\right\}$ and $g: \bar{B} \rightarrow \bar{E}$ is an indirectly conformal (or anticonformal) map from $\bar{B}$ onto $\bar{E}$ such that $g(1,0)=\boldsymbol{o}_{1}, g(-1,0)=\boldsymbol{o}_{2}$ and $g(u, 0) \in \boldsymbol{o}_{1} \boldsymbol{o}_{2}$ for each $u \in[-1,1]$. Notice that $K(u, 0)=\mathcal{O}$ for $u \in[-1,1]$ (see Figure 6). Set 


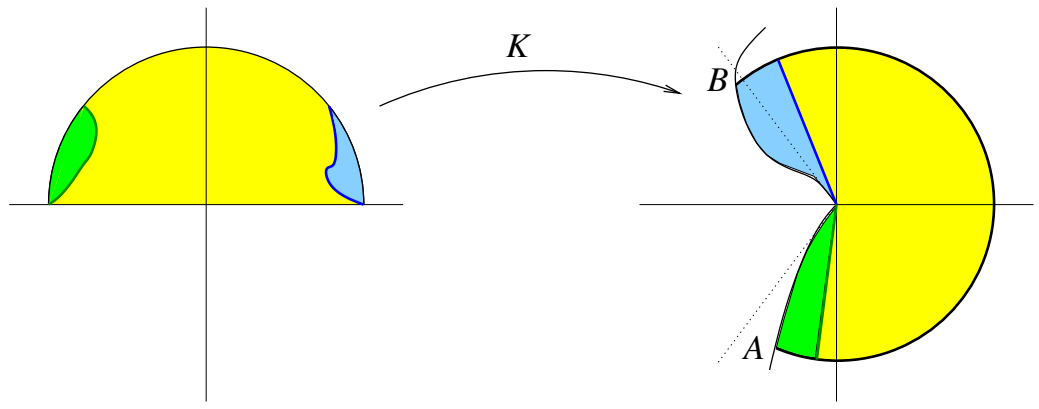

Figure 6. $L\left(\alpha_{2}\right), K^{-1}\left(L\left(\alpha_{2}\right)\right)$ (blue curves); $L\left(\alpha_{1}\right), K^{-1}\left(L\left(\alpha_{1}\right)\right)$ (green curves).

$x=a \circ g, y=b \circ g$ and $z=c \circ g$, so that $X(u, v)=(x(u, v), y(u, v), z(u, v))$ for $(u, v) \in B$. Now, from Step 2 of the proof of Theorem 1 of [Lancaster and Siegel 1996a],

$$
X \in C^{0}\left(\bar{B} \backslash\{( \pm 1,0)\}: \mathbb{R}^{3}\right) \cap C^{1, \iota}\left(B \cup\{(u, 0):-1<u<1\}: \mathbb{R}^{3}\right)
$$

for some $\iota \in(0,1)$ and $X(u, 0)=(0,0, z(u, 0))$ cannot be constant on any nondegenerate interval in $(-1,1)$. Define $\Theta(u)=\arg \left(x_{v}(u, 0)+i y_{v}(u, 0)\right)$. From equation (12) of [Lancaster and Siegel 1996a], we see that

$$
\alpha_{1}=\lim _{u \downarrow-1} \Theta(u) \quad \text { and } \quad \alpha_{2}=\lim _{u \uparrow 1} \Theta(u) ;
$$

here $\alpha_{1}<\alpha_{2}$. As in Steps 2-5 of the proof of Theorem 1 of [Lancaster and Siegel 1996a], we see that $R f(\theta)$ exists when $\theta \in\left(\alpha_{1}, \alpha_{2}\right)$,

$$
\begin{array}{lll}
\overline{G^{-1}\left(L\left(\alpha_{2}\right)\right)} \cap \partial E=\left\{\boldsymbol{o}_{1}\right\} & \text { (and } \left.\overline{K^{-1}\left(L\left(\alpha_{2}\right)\right)} \cap \partial B=\{(1,0)\}\right) & \text { if } \alpha_{2}<\alpha, \\
\overline{G^{-1}\left(L\left(\alpha_{1}\right)\right)} \cap \partial E=\left\{\boldsymbol{o}_{2}\right\} & \text { (and } \left.\overline{K^{-1}\left(L\left(\alpha_{1}\right)\right)} \cap \partial B=\{(-1,0)\}\right) & \text { if } \alpha_{1}>-\alpha,
\end{array}
$$

where $L(\theta)=\left\{(r \cos \theta, r \sin \theta) \in \Omega: 0<r<\delta^{*}\right\}$, and one of the following cases holds:

(a) $R f$ is strictly increasing or strictly decreasing on $\left(\alpha_{1}, \alpha_{2}\right)$.

(b) There exist $\alpha_{L}, \alpha_{R}$ so that $\alpha_{1}<\alpha_{L}<\alpha_{R}<\alpha_{2}, \alpha_{R}=\alpha_{L}+\pi$, and $R f$ is constant on $\left[\alpha_{L}, \alpha_{R}\right]$ and either increasing on $\left(\alpha_{1}, \alpha_{L}\right]$ and decreasing on $\left[\alpha_{R}, \alpha_{2}\right)$ or decreasing on $\left(\alpha_{1}, \alpha_{L}\right]$ and increasing on $\left[\alpha_{R}, \alpha_{2}\right)$.

If $\alpha_{2}=\alpha$ and $\alpha_{1}=-\alpha$, then Theorem 1 is proven. Otherwise, suppose $\alpha_{2}<\alpha$ and fix $\delta_{0} \in\left(0, \delta^{*}\right)$ and $\Omega_{0}$ (see Figure 4$)$ as before in case (i).

Claim. Suppose $\alpha_{2}<\alpha$. Then $f$ is uniformly continuous on $\Omega_{0}^{+}$, where

$$
\Omega_{0}^{+}:=\left\{(r \cos \theta, r \sin \theta) \in \Omega_{0}: 0<r<\delta^{*}, \alpha_{2}<\theta<\pi\right\} .
$$




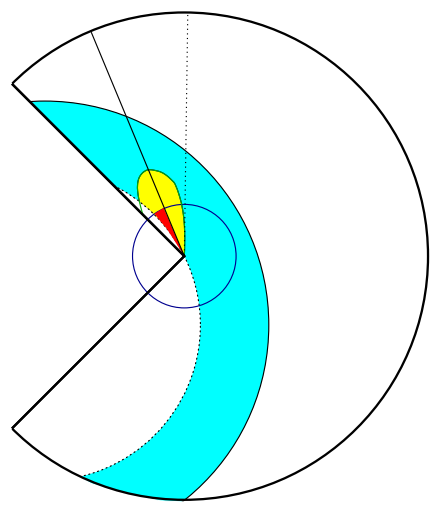

Figure 7. $\Omega^{*} \cap \mathcal{A}\left(\boldsymbol{p}_{1}\right)$ (blue, yellow and red regions), $\partial B_{\eta(\delta)}(\mathcal{O})$ (blue circle).

Proof. Suppose $\alpha-\alpha_{2}<\pi$ (see the blue region in Figure 6). Let $\epsilon>0$. Choose $\delta \in\left(0, \delta_{0}\right)$ such that $p(\delta)+q(p(\delta))<\frac{1}{4} \epsilon$ and $p(\delta)<r_{2}-r_{1}$. Let $C_{r}=\{(u, v) \in \bar{B}:|(u, v)-(1,0)|=r\}$ and let $l_{r}$ be the arclength of the image curve $X\left(C_{r}\right)$. The Courant-Lebesgue lemma implies that for each $\delta \in(0,1)$, there exists a $\rho(\delta) \in(\delta, \sqrt{\delta})$ such that $l_{\rho(\delta)}<p(\delta)$. Denote the endpoints of $C_{\rho(\delta)}$ as $\left(u_{1}(\delta), v_{1}(\delta)\right)$ and $\left(u_{2}(\delta), 0\right)$, where $\left(u_{1}(\delta)\right)^{2}+\left(v_{1}(\delta)\right)^{2}=1, v_{1}(\delta)>0$ and $u_{2}(\delta) \in(-1,1)$. Notice $\Theta\left(u_{2}(\delta)\right)<\alpha_{2}$; let us assume that $\delta$ is small enough that $\alpha-\Theta\left(u_{2}(\delta)\right)<\pi$.

Now $X\left(C_{\rho(\delta)}\right)$ is a curve whose tangent ray at $\mathcal{O}$ exists and has direction $\theta=\Theta\left(u_{2}(\delta)\right)$ and $\partial \Omega \cap X\left(C_{\rho(\delta)} \backslash\left\{\left(u_{1}(\delta), v_{1}(\delta)\right),\left(u_{2}(\delta), 0\right)\right\}\right)=\varnothing$; hence there exists $\eta=\eta(\delta)>0$ such that $\left\{\boldsymbol{x} \in \Omega_{0}^{+}:|\boldsymbol{x}|<\eta(\delta)\right\}$ (the red region in Figure 7) is a subset of $\Omega_{0} \cap X(\{(u, v) \in \bar{B}:|(u, v)-(1,0)|<\rho(\delta)\})$ (the yellow region plus the red region in Figure 7). From (4) and the arguments in the proof of the claim in case (i), we see that $f$ is uniformly continuous on $\Omega_{0}^{+}$.

If $\alpha-\alpha_{2} \geq \pi$, we argue as in the proof of the claim in case (i) and see that $f$ is uniformly continuous on $\Omega_{0}^{+}$.

Thus $f \in C^{0}\left(\bar{\Omega}_{0}^{+}\right)$; hence (5) implies that $R f(\theta)=\lim _{\tau \uparrow \alpha_{2}} R f(\tau)$ for all $\theta \in$ $\left[\alpha_{2}, \alpha\right)$. Suppose $\alpha_{1}>-\alpha$. Then, as above, $f$ is uniformly continuous on

$$
\Omega_{0}^{-}:=\left\{(r \cos \theta, r \sin \theta) \in \Omega_{0}: 0<r<\delta^{*},-\pi<\theta<\alpha_{1}\right\}
$$

and $f \in C^{0}\left(\bar{\Omega}_{0}^{-}\right)$; hence (5) implies

$$
R f(\theta)=\lim _{\tau \downarrow \alpha_{1}} R f(\tau) \quad \text { for all } \theta \in\left(-\alpha, \alpha_{1}\right] .
$$

Thus Theorem 1 is proven. 


\section{Proof of Theorem 2}

The parametric representation (3) with properties $\left(a_{1}\right)-\left(a_{5}\right)$ continues to be valid and either case (A) or case (B) holds true.

Suppose case (A) holds. Let $q_{1}$ denote the modulus of continuity of the trace of $f$ on the (closed) set $\partial^{-} \Omega^{*}$ (i.e., $\left|f\left(\boldsymbol{x}_{1}\right)-f\left(\boldsymbol{x}_{2}\right)\right| \leq q_{1}\left(\left|\boldsymbol{x}_{1}-\boldsymbol{x}_{2}\right|\right)$ if $\left.\boldsymbol{x}_{1}, \boldsymbol{x}_{2} \in \partial^{-} \Omega^{*}\right)$. Fix $\delta_{0} \in\left(0, \delta^{*}\right)$ with $\sqrt{\delta_{0}}<\min \{|\boldsymbol{o}-\boldsymbol{a}|,|\boldsymbol{o}-\boldsymbol{b}|\}$. Let $\boldsymbol{p}_{1} \in \mathbb{R}^{2}$ satisfy $\left|\boldsymbol{p}_{1}\right|=r_{1}$ and $\left|\boldsymbol{p}_{1}-\boldsymbol{y}_{1}\left(\delta_{0}\right)\right|=r_{1}$ such that $\boldsymbol{p}_{1}$ lies above (and to the left of) the line through $\mathcal{O}$ and $\boldsymbol{y}_{1}\left(\delta_{0}\right)$. Set $\Omega_{0}=\left\{\boldsymbol{x} \in \Omega^{*}:\left|\boldsymbol{x}-\boldsymbol{p}_{1}\right|>r_{1}\right\}$.

Claim. $f$ is uniformly continuous on $\Omega_{0}$.

Proof. Let $\epsilon>0$. Choose $\delta \in\left(0, \delta_{0}\right)$ such that $p(\delta)+q(p(\delta))+q_{1}(p(\delta))<\frac{1}{2} \epsilon$ and $p(\delta)<r_{2}-r_{1}$. Pick a point $\boldsymbol{w} \in C_{\rho(\delta)}^{\prime}$ and define $b_{1}^{ \pm}: \mathcal{A}\left(\boldsymbol{p}_{1}\right) \rightarrow \mathbb{R}$ by

$$
b_{1}^{ \pm}(\boldsymbol{x})=f(\boldsymbol{w}) \pm p(\delta) \pm h_{\boldsymbol{p}_{1}}(\boldsymbol{x}), \quad \boldsymbol{x} \in \mathcal{A}\left(\boldsymbol{p}_{1}\right) .
$$

Notice that

$$
b_{1}^{-}(\boldsymbol{x})<f(\boldsymbol{x})<b_{1}^{+}(\boldsymbol{x}) \quad \text { for } \boldsymbol{x} \in B_{\rho(\delta)}^{\prime} \cap \mathcal{A}\left(\boldsymbol{p}_{1}\right) .
$$

Now there exists $\eta=\eta(\delta)>0$ such that $\left\{x \in \Omega_{0}:|x|<\eta(\delta)\right\}$ (the red regions in Figure 8) is a subset of $B_{\rho(\delta)}^{\prime} \cap \mathcal{A}\left(\boldsymbol{p}_{1}\right)$ (the yellow regions plus the red regions in Figure 8). Thus, for $\boldsymbol{x}_{1}, \boldsymbol{x}_{2} \in \Omega_{0}$ satisfying $\left|\boldsymbol{x}_{1}\right|<\eta(\delta),\left|\boldsymbol{x}_{2}\right|<\eta(\delta)$, we have

$$
\left|f\left(\boldsymbol{x}_{1}\right)-f\left(\boldsymbol{x}_{2}\right)\right|<2 p(\delta)+2 q(p(\delta))+2 q_{1}(p(\delta))<\epsilon .
$$

The remainder of the proof of the claim follows as before.

The proof of Theorem 2 in this case now follows the proof of Theorem 1 in the same case.

If case (B) holds, then the proof of Theorem 2 is essentially the same as the proof of Theorem 1; the only significant difference is that $z \in C^{0}(\bar{B} \backslash\{(1,0)\})$ (and $\left.c \in C^{0}\left(\bar{E} \backslash\left\{\boldsymbol{o}_{1}\right\}\right)\right)$ and hence $R f(\theta)$ exists for $\theta \in[-\alpha, \alpha)$.
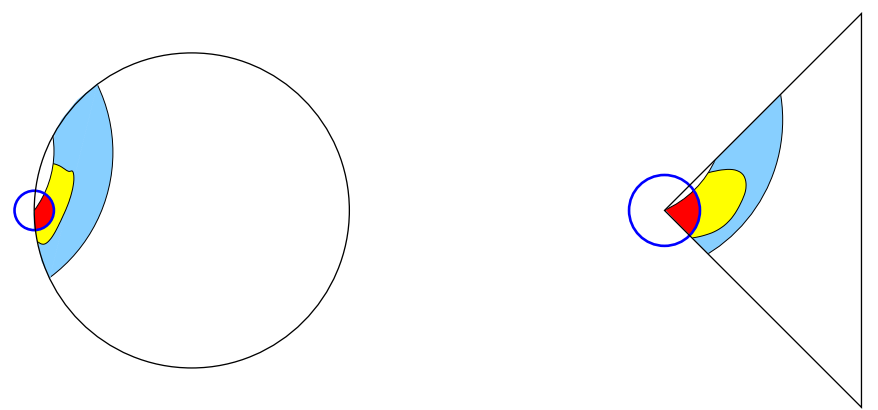

Figure 8. $\Omega_{0} \cap \mathcal{A}\left(\boldsymbol{p}_{1}\right)$ (blue, yellow and red regions), $\partial B_{\eta(\delta)}(\mathcal{O})$ (blue circle). 


\section{References}

[Courant 1950] R. Courant, Dirichlet's principle, conformal mapping, and minimal murfaces, Interscience, New York, 1950. MR 0036317

[Elcrat and Lancaster 1986] A. R. Elcrat and K. E. Lancaster, "Boundary behavior of a nonparametric surface of prescribed mean curvature near a reentrant corner", Trans. Amer. Math. Soc. 297:2 (1986), 645-650. MR 854090

[Jin and Lancaster 1997] Z. Jin and K. Lancaster, "Behavior of solutions for some Dirichlet problems near reentrant corners", Indiana Univ. Math. J. 46:3 (1997), 827-862. MR 1488339

[Lancaster 1985] K. E. Lancaster, "Boundary behavior of a nonparametric minimal surface in $\mathbf{R}^{3}$ at a nonconvex point”, Analysis 5:1-2 (1985), 61-69. MR 791492

[Lancaster 1987] K. E. Lancaster, "Boundary behavior of nonparametric minimal surfaces-some theorems and conjectures", pp. 37-41 in Variational methods for free surface interfaces (Menlo Park, CA, 1985), edited by P. Concus and R. Finn, Springer, New York, 1987. MR 872886

[Lancaster 1988] K. E. Lancaster, "Nonparametric minimal surfaces in $\mathbf{R}^{3}$ whose boundaries have a jump discontinuity”, Internat. J. Math. Math. Sci. 11:4 (1988), 651-656. MR 959444

[Lancaster 1989] K. E. Lancaster, "Existence and nonexistence of radial limits of minimal surfaces", Proc. Amer. Math. Soc. 106:3 (1989), 757-762. MR 969523

[Lancaster 1991] K. E. Lancaster, "Boundary behavior near re-entrant corners for solutions of certain elliptic equations", Rend. Circ. Mat. Palermo (2) 40:2 (1991), 189-214. MR 1151584

[Lancaster and Siegel 1996a] K. E. Lancaster and D. Siegel, "Existence and behavior of the radial limits of a bounded capillary surface at a corner", Pacific J. Math. 176:1 (1996), 165-194. MR 1433987

[Lancaster and Siegel 1996b] K. E. Lancaster and D. Siegel, "Behavior of a bounded non-parametric $H$-surface near a reentrant corner", Z. Anal. Anwendungen 15:4 (1996), 819-850. MR 1422643

[Lancaster and Siegel 1997] K. E. Lancaster and D. Siegel, "Correction to: "Existence and behavior of the radial limits of a bounded capillary surface at a corner"”, Pacific J. Math. 179:2 (1997), 397-402. MR 1452541

Received October 26, 2015. Revised February 13, 2016.

MOZHGAN (NORA) ENTEKHABI

DePartment OF MATHEMATiCs, Statistics AND Physics

WICHITA STATE UNIVERSITY

WICHITA, KS 67260-0033

UNITED STATES

mozhgan.entekhabi@gmail.com

KIRK E. LANCASTER

DePartment of Mathematics, Statistics And Physics

WICHITA STATE UNIVERSITY

WICHITA, KS 67260-0033

UNITED STATES

lancaster@math.wichita.edu 


\title{
PACIFIC JOURNAL OF MATHEMATICS
}

Founded in 1951 by E. F. Beckenbach (1906-1982) and F. Wolf (1904-1989)

$$
\text { msp.org/pjm }
$$

\section{EDITORS}

\author{
Don Blasius (Managing Editor) \\ Department of Mathematics \\ University of California \\ Los Angeles, CA 90095-1555 \\ blasius@math.ucla.edu
}

\author{
Paul Balmer \\ Department of Mathematics \\ University of California \\ Los Angeles, CA 90095-1555 \\ balmer@math.ucla.edu \\ Robert Finn \\ Department of Mathematics \\ Stanford University \\ Stanford, CA 94305-2125 \\ finn@math.stanford.edu \\ Sorin Popa \\ Department of Mathematics \\ University of California \\ Los Angeles, CA 90095-1555 \\ popa@math.ucla.edu
}

\author{
Vyjayanthi Chari \\ Department of Mathematics \\ University of California \\ Riverside, CA 92521-0135 \\ chari@math.ucr.edu \\ Kefeng Liu \\ Department of Mathematics \\ University of California \\ Los Angeles, CA 90095-1555 \\ liu@math.ucla.edu \\ Igor Pak \\ Department of Mathematics \\ University of California \\ Los Angeles, CA 90095-1555 \\ pak.pjm@gmail.com \\ Paul Yang \\ Department of Mathematics \\ Princeton University \\ Princeton NJ 08544-1000 \\ yang@math.princeton.edu
}

\section{PRODUCTION}

Silvio Levy, Scientific Editor, production@msp.org

\section{SUPPORTING INSTITUTIONS}

ACADEMIA SINICA, TAIPEI

CALIFORNIA INST. OF TECHNOLOGY

STANFORD UNIVERSITY

UNIV. OF BRITISH COLUMBIA

UNIV. OF CALIFORNIA, BERKELEY

UNIV. OF CALIFORNIA, DAVIS

UNIV. OF CALIFORNIA, LOS ANGELES

UNIV. OF CALIFORNIA, RIVERSIDE

UNIV. OF CALIFORNIA, SAN DIEGO

UNIV. OF CALIF., SANTA BARBARA
KEIO UNIVERSITY

MATH. SCIENCES RESEARCH INSTITUTE

NEW MEXICO STATE UNIV.

OREGON STATE UNIV.
Daryl Cooper

Department of Mathematics

University of California

Santa Barbara, CA 93106-3080 cooper@math.ucsb.edu

Jiang-Hua Lu

Department of Mathematics

The University of Hong Kong

Pokfulam Rd., Hong Kong

jhlu@maths.hku.hk

$$
\text { Jie Qing }
$$

Department of Mathematics

University of California

Santa Cruz, CA 95064

qing@ cats.ucsc.edu

\author{
UNIV. OF CALIF., SANTA CRUZ \\ UNIV. OF MONTANA \\ UNIV. OF OREGON \\ UNIV. OF SOUTHERN CALIFORNIA \\ UNIV. OF UTAH \\ UNIV. OF WASHINGTON \\ WASHINGTON STATE UNIVERSITY
}

These supporting institutions contribute to the cost of publication of this Journal, but they are not owners or publishers and have no responsibility for its contents or policies.

See inside back cover or msp.org/pjm for submission instructions.

The subscription price for 2016 is US $\$ 440 /$ year for the electronic version, and \$600/year for print and electronic.

Subscriptions, requests for back issues and changes of subscriber address should be sent to Pacific Journal of Mathematics, P.O. Box 4163, Berkeley, CA 94704-0163, U.S.A. The Pacific Journal of Mathematics is indexed by Mathematical Reviews, Zentralblatt MATH, PASCAL CNRS Index, Referativnyi Zhurnal, Current Mathematical Publications and Web of Knowledge (Science Citation Index).

The Pacific Journal of Mathematics (ISSN 0030-8730) at the University of California, c/o Department of Mathematics, 798 Evans Hall \#3840, Berkeley, CA 94720-3840, is published twelve times a year. Periodical rate postage paid at Berkeley, CA 94704, and additional mailing offices. POSTMASTER: send address changes to Pacific Journal of Mathematics, P.O. Box 4163, Berkeley, CA 94704-0163.

PJM peer review and production are managed by EditFLOW ${ }^{\circledR}$ from Mathematical Sciences Publishers.

PUBLISHED BY

\section{I. mathematical sciences publishers}

nonprofit scientific publishing

http://msp.org/

(C) 2016 Mathematical Sciences Publishers 


\section{PACIFIC JOURNAL OF MATHEMATICS}

Volume $283 \quad$ No. $2 \quad$ August 2016

The fundamental theorem of tropical differential algebraic geometry

FUENSANTA AROCA, CRISTHIAN GARAY and ZEINAB TOGHANI

A simple solution to the word problem for virtual braid groups

271

PaOlo Bellingeri, Bruno A. Cisneros de la CruZ and Luis

PARIS

Completely contractive projections on operator algebras

DAVID P. BLECHER and MATTHEW NEAL

Invariants of some compactified Picard modular surfaces and applications

AMIR DŽAMBIĆ

Radial limits of bounded nonparametric prescribed mean curvature surfaces

MOZHGAN (NORA) ENTEKHABI and KIRK E. LANCASTER

A remark on the Noetherian property of power series rings

BYUNG GYUN KANG and PHAN THANH TOAN

Curves with prescribed intersection with boundary divisors in moduli spaces of curves

\section{XIAO-LEI LIU}

Virtual rational Betti numbers of nilpotent-by-abelian groups

BEHROOZ MiRZAII and FATEMEH Y. MOKARI

A planar Sobolev extension theorem for piecewise linear homeomorphisms 405

EMANUELA RADICI

A combinatorial approach to Voiculescu's bi-free partial transforms

PAUL SKOUFRANIS

Vector bundle valued differential forms on $\mathbb{N} Q$-manifolds

LUCA VitAGLiANO

Discriminants and the monoid of quadratic rings 